\title{
The Impact of Macro-Economic Factors on Non-Performing Loans in Albania
}

\section{Professore Associato Anilda Bozdo}

Titolare all'Università Europea di Tirana; anildabozdo@gmail.com

\section{Dr. Ermela Kripa}

Titolare all'Università Europea di Tirana; ermelakripa@gmail.com

\author{
Doi:10.5901/ajis.2015.v4n3s1p534
}

\section{Abstract}

\begin{abstract}
Albania's banking system has made great progress, especially in recent decades. In the last five years the economy has had many important changes, with regard to financial sustainability in Albania. Our country is going through a difficult moment and the Albanian banking system is characterized by high levels of non-performing loans. Our banking system has several problems and the authorities are not giving the proper solution and the implementation of these solutions is taking too much time, thing that aggravates even further the current situation. Our hypothesis in this paper is: despite the totality of all the macroeconomic factors that influence in a country, the main factor that had a negative impact on the high level of nonperforming loans in Albania is the gross domestic product. To prove this hypothesis, we used two methods, the first one is the linear regression and the other one is stress tests. Besides that, we have referred to the most recent literature and see also the experience of other countries. The same methods were also used by other authors both foreign and Albanian such as (2004), Vlieghe (2001) Shijaku \& Czech (2010) etc. Meanwhile, we have also analyzed the internal factors which had an impact on the portfolio of non-performing loans in commercial banks in Albania, highlighting the impact of the professional analysts of credit, the implementation of procedures and manuals from banks, corruption, the rapid growth of the non-performing loans during 2007-08 etc.
\end{abstract}

Keywords: non-performing loans, the banking system, the gross domestic product, macroeconomic factors, corruption.

\section{Introduzione}

L'Albania come tutti gli altri paesi in transizione, ha vissuto tanti fenomeni connessi alla transizione, ed ha anche fatto delle grandi riforme nel sistema finanziario del paese tra le altre la creazione del sistema bancario a due livelli, la Banca Centrale e le banche commerciali. II nostro sistema bancario ha vissuto dei grandi cambiamenti anno dopo anno e il cambiamento più importante e stato quello nell'erogazione dei presiti. Essendo che questo sistema bancario era nuovo si sonno verificati dei fattori che hanno favorito e altri no, i quali hanno inciso più tardi nella creazione dei prestiti con problemi. II fattore più importane potremmo dire era la mancanza dell'esperienza, per il fatto che prima degli anni '90 noi avevamo solo una banca, la banca Nazionale la quale quasi non dava prestiti per gli individui (casi rari solo mutui per la casa). II latto positivo era che noi potevamo sfruttare l'esperienza degli altri paesi per non ripetere gli stessi errori, usando tutta la legislazione bancaria dei paesi occidentali sia quelli della regione per disegnare i regolamenti e le procedure dell'intera attività creditizia, delle trasferte, riciclaggio di denaro sporco etc. Specialmente l'attività creditizia, che era una nuova attività per le banche albanesi, ha avuto molti problemi soprattutto in quelle banche dove lo stato Albanese era uno dei diretti azionisti. Con il processo di privatizzazione il portafoglio di queste banche si pulì, attraverso la concentrazione di questi prestiti con problemi nell' Agenzia del Trattamento dei Crediti (ATC). Con l'ingresso delle banche privati nei primi anni il livello del attività creditizia era basso e la loro qualità non poteva influire nel risultato finanziario delle banche.

Siccome si stavano creando delle nuove banche per la prima volta in Albania si stava creando anche il capitale umano, termine questo usato per la prima volta dall'economista Theodore Schultz, nel 1971. Lui credeva che il capitale umano era come ogni altro tipo di capitale, per il quale si poteva investire nell'educazione, corsi di formazione etc., che avrebbe comportato il miglioramento della qualità del lavoro.

Dopo il 2000, con l'ingresso delle banche con capitale straniero, come Reiffessen Bank, Intesa San-Paolo Bank, 
Societe General Bank, il sistema bancario si sviluppo in maniera lampante e questo sviluppo si e riflesse nella crescita dell'attività creditizia delle banche. Questa attività ha avuto il pico nel 2008 con la crescita del portafoglio prestiti con il $46.77 \%^{1}$ comparato con il mese dicembre del 2007. Dopo quest'anno, come risultato della crisi finanziaria che ha coinvolto tutti i paesi del mondo, si e verificato anche in Albania il processo del calo dell'attività creditizia delle banche, il rapporto del portafoglio crediti nel 2009 comparato con quello del 2008 cadde fino al $14.15^{2}$ per cento. Da quest'anno in poi, il ritmo di crescita di questo portafoglio prestiti e venuto ad abbassarsi fino nel 2013 , anno nel quale abbiamo il primo calo della crescita di questo portafoglio con il -1.1 per cento. Nel 2014 abbiamo un altro rialzo di questo portafoglio con il $4.85^{3}$ per cento.

Le banche in Albania non solo aumentarono il portafoglio dei prestiti, ma aumentarono anche la tipologia dei prodotti bancari che veniva offerta alla clientela come sportelli bancomat, e-banking, POS, carte di credito, carte di debito etc., cosa che ha dato uno sviluppo molto importante all'impresa bancaria in Albania.

Con l'inizio della crisi finanziaria nel 2007 fino ad oggi, anche se in Albania non si e avuta un'influenza diretta, poi che non era quotata nei mercati internazionali, il sistema bancario registro un calo, semplicemente dal panico bancario, cosa ce porto all'abbassamento dei depositi dal settembre del 2008 fino a marzo 2009 con il 9.5 per cento. Nell' aprile del 2009 con l'intervento da parte della Banca Centrale Albanese con l'assunzione di diverse misure a rassicurare il pubblico ed i mercati fece che a dicembre del 2009 si ebbe una crescita del 1.6 percento comparato con il settembre del 2008.

Un'altra caratteristica dello sviluppo del nostro sistema bancario durante questi anni, e stata la crescita del numero delle filiali e delle agenzie in tutto il territorio Albanese. Questo ha fatto sì che i servizi e i prodotti bancari potessero essere offerte anche in quei territori che erano meno sviluppati, quindi attraverso il miglioramento dell'infrastruttura interna delle reti bancarie.

Le banche crescendo e rafforzandosi migliorarono la loro struttura organizzativa, creando dei dipartimenti appositi di gestione del rischio, cosa che prima degli anni 2000 non esisteva, creando il Comitato ALCO etc.

Negli ultimi tempi le nostre banche sono entrate anche nell'attività del leasing, creando le società a loro a carico per sviluppare questa attività.

\section{Un Panorama Sopra il Pensiero Economico Connesso con i Fattori Determinanti del Processo Creditizio delle Banche}

Molti autori hanno studiato il fenomeno negativo dei prestiti con problemi, perché questo e un fenomeno internazionale il quale incide direttamente sui risultati delle banche. Per l'Albania questo e un nuovo dibattito, poiché come lo abbiamo sottolineato sopra noi abbiamo soltanto due decenni di esperienza nel processo creditizio.

Keeton \& Morris (1987) hanno fatto una ricerca in circa 2000 banche negli Stati Uniti, per spiegare il cambiamento del livello dei prestiti con problemi in queste banche. Secondo gli autori alcune banche hanno registrato delle grandi perdite a cause connesse alla casualità, altre dalla cattiva gestione del processo creditizio ed altre ancora hanno avuto la possibilità di creare dei portafogli ben diversificati che hanno permesso a loro di abbassare i criteri per dare dei prestiti e di mantenere un rischio minimo. Un'altra ragione e che alcune banche erano in paesi con un economia non molto sviluppata e le altre erano in paesi con un economia forte. L'argomento di questi autori ci aiuta anche a verificare la nostra ipotesi secondo il quale il PIL e il fattore principale che incide sulla qualità del portafoglio dei prestiti.

La maggior parte delle banche avevano avuto problemi perché quelle erano specializzate a dare dei prestiti solo in un settore dell'economia. Questo porta in una mancata possibilità per diversificare il portafoglio dei prestiti, cioè a diminuire il rischi di credito.

Robert T. Clair (1992) nella sua ricerca conclude che esiste un legame tra la crescita dei prestiti e la sua qualità. Secondo l'autore, per misurare la qualità del prestito si devono usare due standard:

(a) II rapporto dei prestiti perduti con la somma totale dei prestiti

(b) II rapporto dei prestiti con problemi con la somma totale dei prestiti, per il fatto che questi due rapporti rappresentano la probabilità attuale della capacita per pagare i prestiti.

L'autore conclude che una crescita rapida dei prestiti porterebbe un miglioramento di questi indici nel breve termine, pero nel lungo termine quelle potrebbero rappresentare un grande potenziale per diventare prestiti con problemi.

\footnotetext{
${ }_{1}^{1}$ Banca d'Albania, il rapporto Annuale 2006-14

${ }^{2}$ Lo stesso

${ }^{3}$ Lo stesso
} 
Secondo noi questo fenomeno è presente anche in Albania, perché le nostre banche quando la crisi comincio, hanno frenato il processo creditizio, e nel frattempo che i prestiti non crescevano i prestiti con problemi aumentavano. Questi prestiti stanno crescendo anche perché il paese sta passando una situazione difficile economica, come ad esempio l'abbassamento del ritmo di crescita del PIL, ma anche perché i problemi per i pagamenti dei prestiti non si verificano nei primi anni che i prestiti sono erogati dalle banche. Questo succede perché spesso il prestito da se si poteva utilizzare per pagare le rate nei primi anni dell'ammortamento del credito (principalmente i mutui per la casa che sonno a lungo termine occupano il 29.46 per cento del portafoglio prestiti nel 2014 (Banca d'Albania).

L'autore si ferma anche sul problema che la gestione dei crediti deve essere più efficace, per impedire che i prestiti diventino dei prestiti con problemi. Lo studio suggerisce che per la crescita dei prestiti e un fattore determinante la qualità dei prestiti, e gli analisti dei prestiti devono usare un informazione ampia per conoscere meglio i loro clienti. Questa cosa e richiesta anche dalle nostre banche. Nei loro manuali del processo creditizio l'analista deve andare al posto di lavoro del cliente nel momento che la banca dia il prestito, il quale deve disegnare un rapporto sulla sua situazione finanziaria per far parte della file del cliente. Dall'altra parte queste visite si devono ripetere ogni anno dal dipartimento del rischio dopo che i prestiti sono stati dati, per monitorarli.

Fernández de Lis, Martínez Pagés \& Saurina (2000) nella loro ricerca analizzano il comportamento ciclico del prestito bancario, cioè la qualità dei prestiti che si riflette nel rapporto prestiti con problemi/totale dei prestiti la quale si presenta con grandi tendenze cicliche in Spagna. Secondo gli autori questa comporta dei problemi anche da parte delle banche, pero anche da parte delle autorità di vigilanza. Gli autori pensano che questa ciclicità dei prestiti con problemi si collega a questi fenomeni:

- $\quad$ il forte legame tra i prestiti con problemi e il ciclo economico

- l'erogazione di grandi somme di prestiti senza rispettare i criteri, in momenti specifici della crescita economica, le quali cominciano a presentare dei problemi, quando l'economia si abbassa, o peggio ancora va in recessione. Lo stesso fenomeno si e verificato pure in Albania. La grande crescita dell'ammontare del prestito erogato nel periodo 2006-2008 ha fatto che i prestiti con problemi aumentassero dopo il 2010 fino ad oggi.

- la voglia dei manager delle banche, a prendere grandi quote di mercato, senza analizzare bene i prestiti erogati, e ad entrare nei nuovi settori o regioni del paese, dimenticando i risultati delle loro istituzioni.

Khemraj \& Pasha (2005) nella loro ricerca cercano di poter identificare i fattori che incidono sul livello dei prestiti con problemi in Guayana, prendendo in considerazione 6 banche commerciali le quali hanno operato durante il periodo 1994-2004.

Usando un modello di regressione, gli autori prendono come variabile dipendente i prestiti con problemi, e come variabili indipendenti i fattori macro-economici e bancari tali come: la crescita annuale del PIL, il tasso reale d'interesse, il tasso d'inflazione, il tasso reale effettivo di cambio, il rapporto tra il totale dei prestiti e il totale delle attività, il tasso reale d'interesse, la percentuale della crescita dei prestiti delle banche. II risultato del loro studio dimostra che, il legame tra il rapporto prestiti/totale attività e quello con i prestiti con problemi e positivo, ciò significa che queste banche hanno erogato più prestiti, cioè hanno un rapporto prestiti/totale attività e livelli più alti dei prestiti con problemi. II tasso reale d'interesse e in diretta relazione con i prestiti con problemi, pero il loro legame e debole. Questo sì e verificato anche in Albania, quando le banche erogavano prestiti durante 2006-08 i tassi reali di interesse erano più alti che negli anni seguenti, pero il livello dei prestiti con problemi era più basso. Secondo noi, questo legame e debole, perché esiste un Gap tra il tempo in cui si applicano gli interessi e il tempo in qui si ha un effetto nella qualità del portafoglio prestiti, ciò è dovuta al fatto che i clienti soffrono dagli alti tassi d'interesse quando l'economia si ferma. La crescita del livello dei prestiti presenta un legame forte e inverso con i prestiti con problemi ed e importante nel tempo t, t-1 e t-2. II tasso reale effettivo di cambio presenta un forte legame con i prestiti con problemi dimostrando che i prestiti in valuta estera vengono influenzati direttamente dal tasso reale di cambio. II legame tra la crescita del PIL e i prestiti con problemi e inverso, invece per quando riguarda il legame tra l'inflazione e i presiti con problemi esiste una confusione poiché i coefficienti sonno negativi per il tempo t e positivi per il tempo t-1. II legame tra la grandezza della banca e i prestiti con problemi non e significativa dimostrando così che le banche più grandi non e che sonno necessariamente più efficienti nel analizzare i prenditori dei prestiti in comparandoli con le banche più piccole.

Braslinš, G. \& Orlovs, A. \& Braukša I. \& Bulis, A. (2014) Questi autori concludono che alcuni indici del PIL e dei prestiti hanno mostrato una crescita positiva fino al 2007, e questo rapporto era legato al cambiamento del PIL. Nel 2008 il volume dei prestiti cresceva anche se il PIL diminuiva. Basandosi in questi studi empirici il fattore determinante tra le due variabili del PIL e del ammontare erogato del prestito, e il PIL, perché i cambiamenti nell'ammontare del prestito erogato seguono i cambiamenti del PIL. Gli autori concludono che i prestiti dei paesi baltici cominceranno a crescere soltanto quando l'economia di questi paesi cresca nei trimestri successivi e non il contrario, che sono i nuovi prestiti 
erogati che incidono la crescita del PIL.

Per testare l'ipotesi gli autori hanno usato il modello causale di Granger (usando dati trimestrali per il periodo 20052010), concludendo che l'ipotesi non si può verificare per tutti i settori dell'economia. Secondo loro il risultato potrebbe essere diverso per gli stessi settori, in diversi paesi. Questo ci fa capire che il modello usato non comprende tutti i fattori che influiscono sulla sua applicazione. I risultati dimostrano che tutte e due le variabili, sia il PIL che l'ammontare del prestito erogato, dipendono fortemente dal loro valore del trimestre precedente.

La possibilità che le imprese possono usare tutte le fonti del prestito è importante sia per il loro sviluppo, nei diversi settori dell'economia sia per la crescita del PIL, pero, dall'altra parte i settori più sviluppati dell'economia hanno più possibilità ad usare i prestiti come fonte di finanziamento. Questo fenomeno si verifica anche nelle banche albanesi, le quali sonno più orientate a dare dei prestiti nei settori più forti dell'economia, come ad esempio il settore dell'edilizia, perché esisteva una maggiore possibilità di pagare i prestiti in questi settori grandi che in altri settori dell'economia, tale come l'agricoltura.

Boudriga, Taktak dhe Defi (2009) Questi autori hanno esteso la loro ricerca in 59 paesi per vedere meglio gli effetti dei fattori che incidono sui prestiti con problemi. Questa ricerca si e estesa nel periodo 2002-2006, studiando non solo tutti i fattori che incidono su di esse, ma hanno preso in considerazione anche il ruolo della Vigilanza della Banca Centrale. I risultati della ricerca dimostrano che gli elementi più importanti che fanno abbassare il livello dei prestiti con problemi sonno: I'alto livello di capitalizzazione, una politica prudenziale delle provvigioni, il concentramento dei prestiti in un solo settore dell'economia ed anche la presenza del capitale straniero. Loro concludono che la partecipazione dello stato nelle banche fa crescere il livello dei prestiti con problemi.

Dopo l'analisi fatta, tramite questa ricerca, gli autori arrivano a dare alcune conclusioni importanti:

a. Quando più alto sia il livello di capitalizzazione bancario, tanto più bassa sarà l'esposizione al rischio di credito.

b. I paesi sviluppati che hanno un economia forte sonno meno rischiati nei confronti degli altri paesi in via di sviluppo perché hanno un sistema economico e bancario più consolidato.

c. La proprietà privata e la partecipazione dei capitali esteri fa sì che i sistemi finanziari siano più sani nelle economie meno sviluppate, mentre nei paesi sviluppati i capitali esteri comportano dei problemi più grandi per i prestiti.

d. Per avere un esposizione più bassa del rischio di credito dobbiamo avere delle leggi più forti, istituzioni più efficaci, controlli più rigorosi e spessi sulle banche.

Cappiello, L., Kadareja, A., Sørensen, Ch. K., Protopapa, M. (2010) Nella loro ricerca testano l'ipotesi vista dal latto opposto, cioè che le fluttuazioni del ammontare dei prestiti e li standard bancari di quest'ultimi incidono sul PIL. Gli autori hanno adottato il modello Driscoll (2004) usato per gli Stati uniti, per misurare l'effetto del prestito bancario nel PIL per l'eurozona. II modello e una forma dell'IS-LM e usa tre equazioni, quello della domanda per le merci, denaro e credito ma anche l'equazione dell'offerta dei prestiti bancari.

I resultati datti dagli autori dimostrano che la crescita reale del prodotto (la variabile endogeno) e un processo auto regressivo perché i suoi valori di uno e due periodi precedenti sono statisticamente significativi. La conclusione degli autori e che diversamente dagli Stati Uniti, i cambiamenti nell'offerta del credito bancario per le imprese non hanno un impatto significativo nell'attività reale dell'economia. Da tutto ciò è importante capire l'importanza che hanno le banche centrali nel monitoraggio dello sviluppo del credito bancario e delle loro politiche.

Hoti llir (2010) Questo autore ha studiato l'impatto dell'esposizione del rischio nel settore bancario in Albania e tratta il problema dell'informazione asimmetrica che caratterizza tutti gli elementi sistemici in questo settore, in Albania. L'autore conclude che l'esame diagnostico copre l'incoerenza tra la Basilea II, IFRS e i Regolamenti della Banca Centrale Albanese, per identificare i rischi del aprovviggiamento del portafoglio crediti nella sua misura e gestione.

L'autore ha sviluppato un modello per misurare l'impatto di stress testing e l'implementazione di IFRS 39 sulla qualità del portafoglio crediti, almeno su base mensile. Il quadro del stress test per il capitale minimo richiesto è basato in uno scenario che tiene conto di tutte le componenti d'esposizione rispettando Basilea II e IFRS. Questo include tutti i paradossi che provengono dallo sviluppo del rischio di credito e di conseguenza, per misurare gli effetti sul capitale minimo richiesto. Questa ricerca fornisce punti di vista simili e concorrenziali nel sistema bancario Albanese e pone l'accento sull'importanza dei modelli simili fra i paesi.

La conclusione più importante data dell'autore per l'Albania, è che non esiste una protezione per i consumatori nei confronti dei prodotti bancari, sia dei depositanti o dei prenditori di prestito. Questo mette in pericolo i depositanti che possono perdere il loro potere d'acquisto, poiché i loro depositi non sono protetti dall'inflazione. 


\section{Un Breve Panorama del Processo Creditizio in Albania}

In generale, la Banca d'Albania con le normative emanate, ha gestito in modo conservatorio il portafoglio delle banche, esercitando il controllo almeno una volta l'anno, per le banche classificate secondo il sistema CAMMELS da 3-5 e una volta in un anno e mezzo per quelle banche classificate 1 e 2 (cioè, molto buone e soddisfacente).

II rischio di credito rappresenta il rischio principale per il sistema bancario. Negli ultimi anni, è stato osservato che tale rischio costituisce un problema molto serio per le banche operanti in Albania. Su questo rischio hanno influenzato fattori interni, dei quali possiamo menzionare:

- La ragione più importante è la corruzione che esiste nel sistema bancario, un fenomeno questo, al quale non e stato dato una grande attenzione, pero il nostro pensiero e che questo e un fenomeno molto diffuso.

- Inadeguato livello professionale degli analisti dei prestiti;

- Il sistema bancario e relativamente nuovo nel processo creditizio e noi sappiamo che i prestiti non dimostrano subito i loro problemi nei primi anni della loro emissione;

- L'espansione che ha avuto l'emissione dei prestiti nel periodo 2006-2008, l'accelerazione delle banche a concedere prestiti per occupare tutti i segmenti del mercato, si sarebbe sicuramente accompagnata dalla crescita dei clienti con problemi, e la mancanza delle capacita umane e dell'infrastruttura interna delle banche.

- Le banche non hanno rispettato in modo rigoroso le procedure e il regolamento sviluppatesi da loro, che in questo periodo hanno lavorato con un certo liberismo nella concessione dei prestiti, senza tuttavia sentire il peso dei prestiti con problemi. (Bozdo, A. \& Testa, M. 2014)

Oltre a questi fattori interni, un impatto significativo ha avuto anche la crisi finanziaria e il declino del ritmo di crescita del PIL, per questo motivo lo scopo del nostro lavoro è quello di sottolineare l'importanza dei fattori macroeconomici sul livello dei prestiti con problemi (NPL, non performing loans) e identificare il principale fattore macroeconomico con effetto negativo.

Grafico 1: II trend dei prestiti 2006-2014

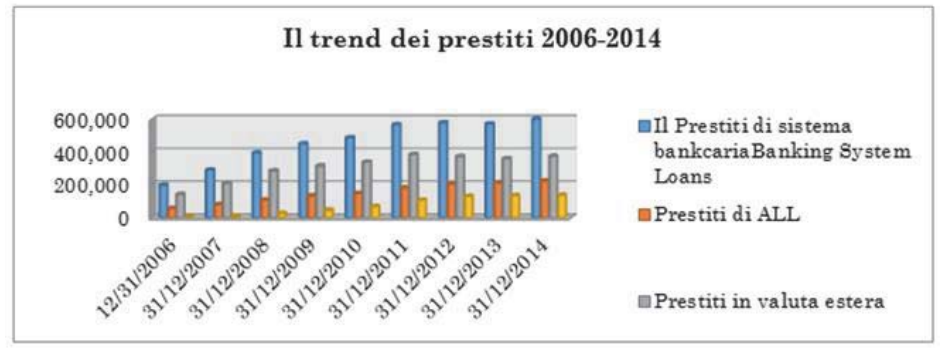

Banca d'Albania, 2006-14

Questo peggioramento della situazione del portafoglio prestiti si è ulteriormente aggravata a causa del fatto che l'economia ha avuto i suoi problemi, il che ha portato al rallentamento della crescita del PIL. 
Grafico 2: PIL e la sua crescita nel arco temporale 2009-135

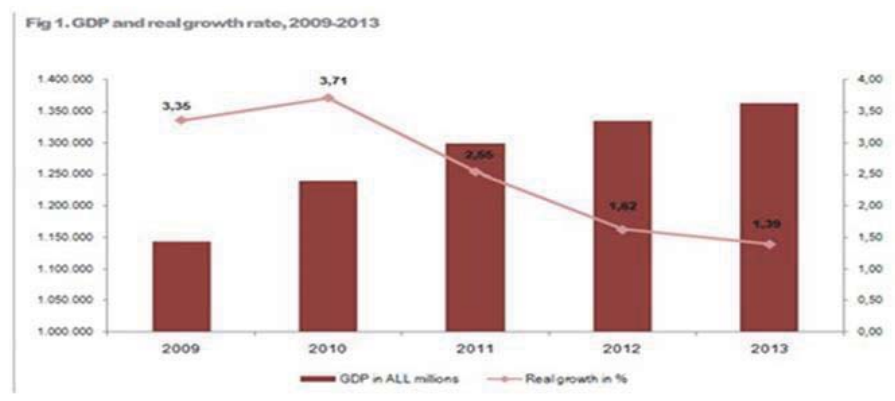

\section{Metodologia e Ipotesi dello Studio}

Per realizzare l'analisi dei fattori macroeconomici abbiamo utilizzato due metodi: il metodo della regressione lineare multipla e lo stress-test. Quest'analisi ha lo scopo di identificare quali fattori macroeconomici operanti in un paese costituiscono il principale fattore che ha influenzato negativamente l'alto livello dei prestiti con problemi in Albania. Un altro scopo di questo studio è quello di vedere come reagisce una variabile macroeconomica quando si fa il test, utilizzando l'analisi di stress-test. Lo stress-test è un'analisi che dimostra la stabilità economica di un soggetto, una società, un istituto finanziario o l'economia intera di un paese.

Per condurre I'analisi attraverso lo stress-test abbiamo costruito un modello per l'Albania, in cui sono stati analizzati tutti gli elementi. In questa parte della ricerca sono stati analizzati gli effetti che hanno sul tasso dei prestiti con problemi, gli indicatori macroeconomici presi in considerazione. Abbiamo poi, costruito un grafico illustrativo della performance del modello per il nostro paese. L'ultima parte dell'analisi comincia con la determinazione dell'arco temporale preso in esame e poi si fa il test di questi valori sotto i tre diversi scenari presi in considerazione.

Il primo scenario è costituito da valori più estremi storici, le quali fanno crescere i prestiti con problemi, li secondo scenario utilizza due deviazioni standard per testare tutte le variabili presi in esame, mentre il terzo scenario comprende le variabili testate attraverso la consultazione con gli esperti. Questi dati testati saranno integrati nel modello al fine di prendere i valori estremi dei prestiti con problemi secondo i scenari di cui sopra menzionati. In seguito abbiamo sviluppato l'analisi del modo in cui reagirebbero i prestiti con problemi per ciascuno dei tre scenari per l'Albania.

\subsection{Limiti della Ricerca}

Uno dei limiti di questo lavoro è che l'analisi non tiene conto del periodo pre-crisi, cioè il periodo 2005-2008. La raccolta dei dati per il periodo prima della crisi era o impossibile o limitata per tutte le variabili necessarie per il test del modello. II nostro lavoro prende in considerazione i dati trimestrali 2008-2014. II fatto che la nostra analisi includa solo gli ultimi 26 trimestri, testimonia la mancanza dei dati su un lungo periodo di tempo. Un altro problema e la mancanza di studi analoghi per l'Albania. Gli studi e gli test di questi modelli vengono realizzati dalle banche centrali, ma non vengono pubblicati come letteratura teorica oppure empirica.

\subsection{La Problematica}

Per realizzare il modello di stress si deve prima costruire un modello che spiega la volatilità del livello dei prestiti con problemi. Tale modello ci aiuta ad analizzare quali sono i fattori macroeconomici che influenzano i prestiti con problemi in Albania. Sulla base della revisione della letteratura sono stati considerati alcuni variabili indipendenti, e la loro significanza è stata testata dal modello. Nel proseguire, noi testeremo la qualità del modello nel suo complesso per il nostro paese.

II prossimo passo della modellazione del comportamento dei prestiti con problemi è quello di evidenziare come 
reagirà il livello di questi prestiti dopo i shock macroeconomici dei scenari predefiniti. Attraverso questa analisi vogliamo vedere se la nostra economia sarà in grado di reagire a queste situazioni macroeconomiche problematiche. Cioè così noi possiamo vedere in che misura aumenterà il livello dei prestiti con problemi nel caso in cui si verifichino delle crisi macroeconomiche.

\subsection{L'ipotesi della Ricerca}

L'ipotesi posta da noi in questo lavoro è: nonostante la totalità di tutti i fattori macroeconomici che influiscono in un paese, il fattore principale che ha inciso negativamente sul alto livello dei prestiti con problemi in Albania e il prodotto interno lordo.

\subsection{I dati}

I dati sui prestiti con problemi sono stati presi dalle pubblicazioni della Banca Centrale dell'Albania e il Ministero delle Finanze. Le percentuali dei prestiti con problemi (NPL) sono stati calcolati per intervalli trimestrali per il periodo compreso tra, il primo trimestre del 2008 al quarto trimestre del 2014. La serie dei prestiti con problemi e la variabile dipendente del modello. I dati macroeconomici sono i variabili indipendenti del modello sono stati presi dalle pubblicazioni dell'Istituto di Statistica Albanese e la Banca Centrale dell'Albania per lo stesso periodo, dal primo trimestre 2008 al primo trimestre 2014.

Riferendosi agli autori Beck, Roland., Jakubik, Peter., \& Pilou, Anamaria (2013) uno dei fattori presi in considerazione per vedere l'impatto sul livello dei prestiti con problemi è stato il tasso reale di scambio EUR/ALL (KR). Per modificare questa variabile viene utilizzato il tasso di cambio nominale e il livello d'inflazione dell'Albania e d'Italia per lo stesso periodo. Abbiamo preso in considerazione l'inflazione dell'Italia per la ragione che questo paese occupa la quota maggiore nella bilancia commerciale del nostro paese, vale a dire che la più grande quantità di importazioni e esportazioni sono verso di essa. In particolare le importazioni dall'Italia nel nostro paese dal 2005-2009 in media compongono il $27 \%$ del totale delle importazioni, mentre le esportazioni verso l'Italia per lo stesso periodo in media compongono il $67 \%$ delle esportazioni totali. In seguito, per modificare questa variabile utilizzeremo la formula seguente:

$$
\begin{aligned}
& K R=r \times \frac{1+p_{I t}}{1+p_{A l}} \\
& \text { Dove: } \\
& K R \rightarrow \text { Tasso Re ale dicambio } \\
& r \rightarrow \text { Tasso No min ale } \\
& p_{I t} \rightarrow \text { L'inf lazione d' Italia } \\
& p_{A l} \rightarrow L^{\prime} \text { 'inf lazione d' Albania }
\end{aligned}
$$

Molti altri autori hanno fatto delle ricerche per il rischio di credito e dei fattori macroeconomici usando il stress test per vedere l'impatto che essi hanno sul prodotto interno lordo. Gli autori Pesola (2001), Virolainen, Kimmo (2004), Havrylchyk (2010), e Vlieghe (2001) hanno considerato l'impatto del PIL sul livello dei prestiti con problemi.

Il livello dei prestiti per l'economia e stata inclusa nell'analisi empirica dei prestiti con problemi da molti autori, tali come: Pesola (2001), Virolainen, Kimmo (2004) e Vlieghe (2001). Il livello dei prestiti per l'economia sarà considerato come il rapporto tra il livello di prestiti del paese e prodotto interno lordo per i rispettivi periodi.

Un altro fattore macroeconomico che sarà preso in considerazione per spiegare la volatilità del livello dei prestiti con problemi, è anche l'inflazione.

Un altro fattore che viene preso in considerazione per l'analisi della volatilità dei prestiti con problemi è il tasso di interesse sui prestiti.

Cioè, per riepilogare possiamo dire che i fattori macroeconomici che vengono presi in considerazione come fattori che incidono sulla capacita dei debitori a essere solventi sono i tassi di interesse, i tassi reali di cambio, il prodotto interno lordo, l'inflazione e il livello del prestiti per l'economia.

\subsection{La costruzione del modello per l'Albania}

Facendo riferimento alla letteratura, si è cercato di analizzare l'importanza che hanno le variabili macroeconomici per spiegare la volatilità del livello dei prestiti con problemi in Albania. Le variabili macroeconomiche prese in considerazione sono: il livello dei prestiti con problemi (NPL) e la variabile dipendente e gli altri cinque fattori macroeconomici sono 
variabili indipendenti: il prodotto interno lordo (PIL), il tasso di interesse sui prestiti (NI), l'inflazione (INF), il livello totale dei prestiti (CER) e il tasso reale di cambio Euro/Lek (KR).

Dopo numerose prove circa l'importanza dei fattori macroeconomici presi in considerazione, abbiamo visto che le variabili indipendenti, tali come il tasso l'interesse e d' inflazione non erano statisticamente significativi nel modello. Alla fine, il modello viene modificato e prende in considerazione solo le seguenti variabili:

$$
N P L=c+\beta_{1} K R_{t-2}+\beta_{2} G D P_{t-1}+\beta_{3} K R E_{t-2}+\varepsilon
$$

Per quanto riguarda il tasso d'interesse, noi pensiamo che non e statisticamente significativo, perché l'effetto che questo tasso ha nel livello prestiti nei paesi come causa e basso. II tasso d'interesse più basso e più attrattivo nei confronti dei riceventi di questi prestiti poiché il prezzo dei prestiti risulterà ad essere più basso. In base a questa deduzione, l'abbassamento del tasso d'interesse dovrebbe influenzare la crescita dei prestiti nel paese. In molti paesi il tasso d'interesse viene usato come meccanismo dalle Banche Centrali per promuovere i prestiti, ma nel nostro paese questo non ha prodotto gli effetti desiderati anche se la Banca Centrale ha abbassato tante volte il tasso base d'interesse al minimo storico. La crescita dei prestiti con problemi nel nostro paese mostra che l'economia risulta ad avere dei problemi (Maho, 2014).Per analizzare l'influenza della volatilità del tasso d'interesse abbiamo disegnato i grafici sottostanti. Come si vede dal grafico 3 l'abbassamento del tasso d'interesse non ha effetto nel innalzamento dei prestiti per l'economia, poiché il livello dei prestiti ha un andamento al ribasso anche se i tassi d'interessi hanno lo stesso andamento, a ribasso. Questa dimostra che il tasso d'interesse non ha influenzato il livello dei prestiti per l'economia. Una possibile spiegazione a questo fenomeno potrebbe essere la crisi economica, o il regresso della domando per prestiti anche se nel nostro paese si verifica un continuo abbassamento del tasso d'interesse.

Grafico 3. L'impatto del tasso d'interesse nel livello dei prestiti nell'economia Albanese

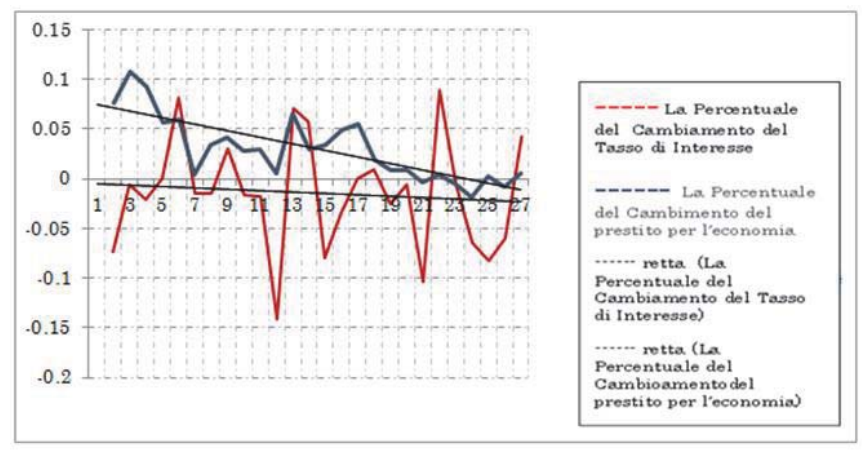

Fonte: Banca d'Albania, 2007-14, Grafico degli autori.

Più in basso nella tabella 1 abbiamo rappresentato alcune statistiche delle variabili descrittive macroeconomiche introdotte nel modello.

Tabella 1. Le statistiche descrittivi dei dati per l'Albania

\begin{tabular}{|l|l|l|l|l|l|}
\hline Le Variabilif & SICLA & Media & Deviazione standard & Minimo & Massimo \\
\hline Tasso Reale of Cambio & KR & 135,102 & 7,398 & 121,152 & 146,853 \\
La Grescita del PIL. & GDP & 0,018 & 0,111 & $-0,130$ & 0,205 \\
I prestifi per Leconomia & KRE & 1,224 & 0,164 & 0,909 & 1,520 \\
\hline
\end{tabular}

\subsubsection{Conclusioni dell'analisi}

Dal punto di vista metodologico in questa ricerca abbiamo già accennato che il metodo che sarà usato per fare l'analisi sarà la regressione lineare multipla. Dopo aver applicato questo metodo, i risultati empirici del modello sono elencati come segue. 
Tabella 2. I risultati Empirici del modello

\begin{tabular}{||l|c|c|c|c||}
\hline \multirow{2}{*}{ Le variabili } & \multirow{2}{*}{ Acronimo } & \multicolumn{3}{|c||}{ Caso del Albania } \\
\cline { 3 - 5 } & & Lag & Coefficiente & Deviazione standard \\
\hline Constante & & - & NA & - \\
\hline Tasso di Interessi & NINT & - & NA & - \\
\hline Tasso Reale di Cambio & KR & -2 & $6.1^{\text {*** }}$ & 1,07 \\
\hline PIL & GDP & -1 & $-1.59^{\star \star *}$ & 0,42 \\
\hline Crediti per l'economia & KRE & -2 & $3.7^{* \star *}$ & 0,31 \\
\hline Livello dei prestiti con problemi & NPL & - & NA & - \\
\hline \hline
\end{tabular}

Nella tabella nr 2. Abbiamo rappresentato il risultato del modello della regressione lineare che ci spiega quali sono i fattori che incidono al cambiamento dei prestiti con problemi. II modello risulta essere statisticamente importante con un livello di confidenza oltre il 99 percento e il coefficiente R2 che spiega l'effetto dei cambiamenti dei prestiti con problemi e al livello del 80 percento. La regressione è stata realizzata dal quarto trimestre del 2007 fino all'ultimo trimestre del 2014, per l'Albania, quindi in tutto 26 osservazioni. Le differenze lag sono state calcolate basandoci nella loro determinazione fatto dagli altri autori, i quali hanno usato questi variabili nei loro lavori, adattandosi all'importanza del modello.

Come possiamo notare dalla tabella 2, il fattore del tasso reale di cambio ha un effetto positivo nell'aumento dei prestiti con problemi. Questo e tipico dei paesi importatori poiché e collegato al fatto che la svalutazione del Lek nei confronti dell'euro porterebbe all'aumento dei prezzi delle merci importate. Per le imprese albanesi che la maggior parte della materie prime le importano, aumenterebbero i loro costo se il Lek si svalutasse nei confronti dell'euro, per questo la probabilità di perdere la capacita di essere solventi per i pagamenti delle rate sarebbe più grande. Ancor di più per $\mathrm{i}$ prestiti in euro questo effetto sarebbe diretto, ciò significa che le rate dei prestiti in euro sarebbero più care se il Lek si svalutasse nei confronti dell'euro. II coefficiente vicino (KR) mostra che il rafforzamento del Lek di 1 percento aumenterebbe il livello dei prestiti con problemi di 6.14 percento dopo un periodo di tre mesi. II tasso d'interesse ha un influenza negativa nella volatilità dei prestiti con problemi.

Altri autori che hanno compreso questa variabili nella loro analisi, sono giunti alla conclusione che l'innalzamento del tasso d'interesse incide nella crescita prestiti con problemi. Secondo loro l'innalzamento del tasso d'interesse farebbe sì che il debito da parte dei quelli che hanno ricevuto il prestito diventasse più caro, incidendo direttamente nella loro capacita di pagamento.

La variabile indipendente, il PIL, secondo il risultato della regressione incide negativamente nel livello dei prestiti con problemi. Questo influenza negativa e sostenuta anche dalle conclusioni simili degli altri autori. II livello dei prestiti con problemi si abbasserebbe nel caso dellinnalzamento del PIL per il fatto che la ripresa economica testimonia un miglioramento della performance delle imprese nel paese, e questa crescita della performance si rifletterebbe in maniera diretta nella loro solvibilità. Quindi il fallimento delle imprese e più ridotto se ce una ripresa economica, la quale e a testimonianza del miglioramento della loro performance. Dai risultati del modello utilizzato ci risulta che la crescita del 1 percento del PIL influenzerebbe in maniera positiva abbassando il livello dei prestiti con problemi nel paese di 1,59 per cento.

Facendo riferimento ai risultati del modello vediamo che i prestiti per l'economia incidono positivamente sui prestiti con problemi. La variabile del prestito per l'economia si calcola come rapporto dei prestiti totali sul PIL del paese. Possiamo dire che quando i prestiti per l'economia crescono, la probabilità che una parte di esse diventino dei prestiti con problemi e maggiore a causa della crescita di quest'ammontare di prestiti. Quando un economia si caratterizza da un alto livello di prestiti, e in caso in cui si verifichi una crisi economica, questo fa sì che le imprese di quest' economia fossero più esposte alla perdita della loro solvibilità. Dalla tabella 2. Possiamo notare che per quanto riguarda l'Albania una crescita ad 1 percento dei prestiti nel paese inciderebbe sul livello dei prestiti con problemi crescendolo con 3.7 percento.

\subsubsection{La Diagnostica del modello}

Per assicuraci dell'esattezza dei risultati prodotti dal modello abbiamo fatto alcune prove che analizzano la qualità del modello.

In primo luogo, si e studiato l'autocorrelazione del modello. Dopo aver fatto il test di Breusch-Godfrey la LM ci risulta che il modello non ha autocorrelazione, perché il valore del Prob.Chi-Square risulta pari al 33 percento, cioè maggiore del 5 percento. (Appendice). 
- In secondo luogo, abbiamo studiato se il modello presenta o meno eteroschedasticità. Dopo il test di BreuschPagan-Godfrey siamo arrivati alla conclusione che il modello non rappresenta eteroschedasticità, poiché il valore del Prob.Chi-Square risulta pari al 22 percento, cioè maggiore del 5 percento. (Appendice).

- In terzo luogo, abbiamo analizzato i residui. Dopo il test della dispersione normale ci risulta che i residui presentano una dispersione normale, poiché il valore del Prob.Chi-Square risulta pari al 22 percento, cioè maggiore del 5 percento. (Appendice).

\subsubsection{La Performance del Modello}

In questa parte viene illustrato graficamente la performance del modello costruito. Più in basso nella tabella 3. e rappresentato l'arco temporale dei dati utilizzati nel modello.

Tabella 3. II periodo di tempo preso in considerazione

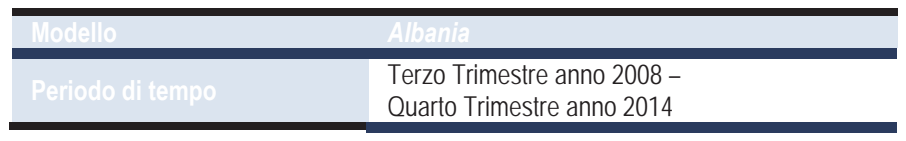

Grafico 4. La performance dei modelli dei prestiti con problemi per l'Albania

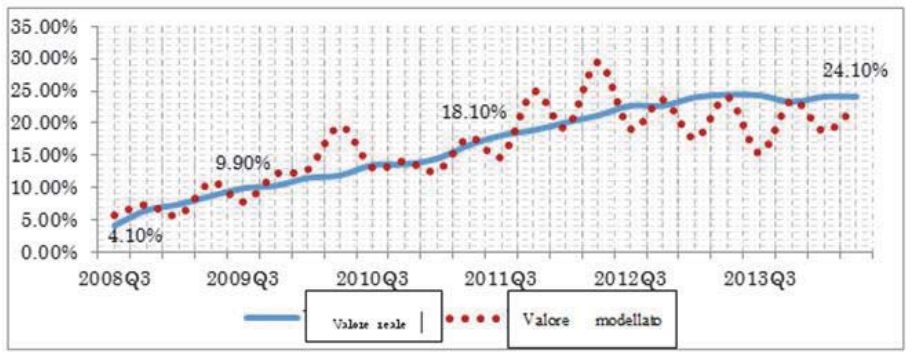

Fonte: Banca d'Albania, Grafico degli autori.

Nel grafico nr. 4 viene illustrato la performance del modello dei prestiti con problemi per l'Albania. Come possiamo vedere dal grafico il livello dei prestiti con problemi ha un andamento crescente durante tutto il periodo di tempo dell'osservazione, che va dal 4,1 percento nel terzo trimestre del 2008 fino al 24,1 percento nell'ultimo trimestre del 2014. Possiamo dire che il modello si avvicina al andamento crescente dei prestiti con problemi anche se non la stessa esattezza. Pero dobbiamo sottolineare che il periodo di tempo preso in considerazione non e molto ampio, per questa ragione questo modello rappresenta dei limiti.

\subsection{Scenari dello Stress-Test}

Una parte molto importante delle prove dello stress-test e la scelta dei scenari. Come accennato già da prima, noi abbiamo analizzato tre scenari (Havrylchyk, 2010). I scenari vengono illustrati più in basso:
I. II peggiore scenario storico
II. Lo scenario con un cambiamento con due deviazioni standard dal valore attuale.
III. L'ultimo scenario basato sul pensiero degli esperti. 
Tabella 4. Le Variabili del Stress-Test

\begin{tabular}{|c|c|c|c|}
\hline & Scenario 1 & Scenario 2 & Scenario 3 \\
\hline Tasso d'lnteresse & NA & NA & NA \\
\hline Tasso Reale di Cambio & 146,85 & 151,75 & 140,75 \\
\hline II Prodotto Interno Lordo & $-0,13$ & $-0,22$ & $-0,18$ \\
\hline I Prestiti per l'Economia & 1,52 & 1,62 & 1,65 \\
\hline It Livello dei Prestific con Problemi & NA & NA & NA \\
\hline
\end{tabular}

Questi scenari verranno integrati nel modello costruito per il caso dell'Albania. Ciascuno delle variabili indipendenti sarà stressato in modo che incidono nella crescita del PIL. Cioè, se la crescita della variabile indipendente influisce nella crescita dei prestiti con problemi, questa variabile crescerà in maniera tale da poter avere la massima incidenza negativa possibile sui prestiti con problemi. In caso contrario la crescita della variabile indipendente abbasserà il livello dei prestiti con problemi. Per vedere in maniera chiara questi cambiamenti delle variabili abbiamo costruito la tabella nr. 4.

\subsection{L'analisi dello Stress Macroeconomico}

Più in basso nella tabella 5 si evidenziano i cambiamenti che subiscono i prestiti con problemi in tutti e tre i scenari. Dopo aver compreso i valori stressati nel modello i loro risultati sono elencati nella tabella più in basso.

Tabella 5. La tabella dei periodi dei stress economici

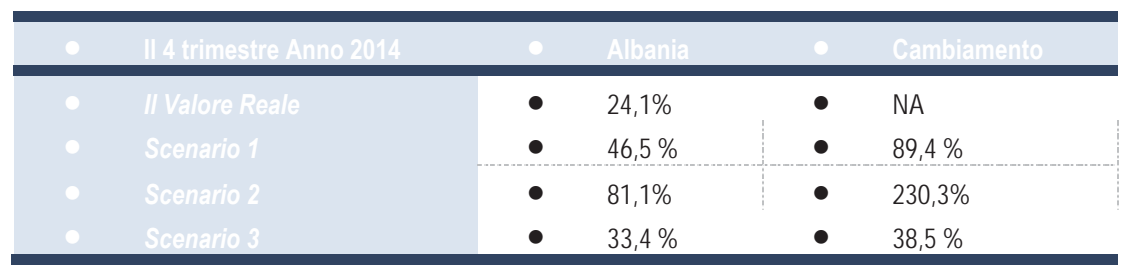

Come si può notare dalla tabella qui sopra, nel caso del primo scenario, cioè di quello dei valori storici più estremi le quali incidono nella crescita dei prestiti con problemi, questo tasso per l'Albania aumenterebbe con il 89,4 percento. Questo impatto cosi grande indica che il tasso dei prestiti con problemi viene influenzato molto dai shock macroeconomici. Da questo si capisce che l'economia albanese non e in grado di ammortizzare bene le crisi macroeconomiche, di conseguenza, il livello dei prestiti con problemi riflette in maniera evidente le variabili stressate che spiegano la sua volatilità.

Nello stesso modo anche il secondo scenario non riflette nessun cambiamento per quanto riguarda l'impatto sul livello dei prestiti con problemi mostrando una crescita ancora più grande del $230,3 \%$ dei prestiti con problemi. Si vede chiaro che la nostra economia e debole e non e capace ad affrontare bene questi shock macroeconomici.

Secondo il terzo scenario, si vede che il cambiamento del livello dei prestiti con problemi e piccola, cioè basandosi sul pensiero degli esperti, possiamo dire che le fluttuazioni sono basse. Per concludere possiamo dire che l'Albania non ha un economia pronta ad ammortizzare gli effetti negativi delle variabili macroeconomiche. Ciò significa che il livello dei prestiti con problemi si influenza molto dal peggioramento di questi variabili macroeconomiche dell'economia Albanese.

Dai risultati qui sopra, possiamo arrivare alla conclusione che per le situazioni più vicine alla realtà, cioè il primo e il terzo scenario, il livello dei prestiti con problemi e molto instabile, invece per i scenari più vicini alla realtà, il secondo scenario, il livello dei prestiti con problemi per l'Albania e più stabile.

\section{Conclusioni e Raccomandazioni}

L'Albania e un paese che si caratterizza da un alto livello del tasso dei prestiti con problemi. Questa situazione problematica, in primo luogo e un indice che dimostra che la nostra economia non e performante e che aumenta la 
possibilità di non pagare le rate dei prestiti. Dall'altra parte le banche, le quali hanno un livello basso del ritorno dei prestiti, rifletteranno questo effetto nel abbassamento del reddito della loro attività. Questo non solo a causa del mancato pagamento dei prestiti, ma anche dalla crescita dei provvigioni le quali peggiorano il risultato finanziario dell'istituzione. L'aumento dei prestiti con problemi in questi ultimi 5 anni ha avuto un andamento crescente con all'incirca il 4 percento l'anno. Nel momento in cui le banche sentono il peso dei prestiti con problemi nei loro bilanci, cominciano a restringere, oppure diventano più caute nel erogare nuovi prestiti. Pero senza il giusto livello di liquidita un economia non e performante. La teoria economica ci dice che c'è sempre bisogno per l'economia di une paese ad avere un livello adeguato di liquidita. La Banca d'Albania durante tutto questo periodo ha cercato di stimolare il livello dei prestiti nel paese, abbassando tante volte il tasso base d'interesse, pero sembra che questa politica non ha prodotto l'effetto desiderato. I diversi fattori che possono impedire la crescita del livello dei prestiti possono essere in tanti, tali come: la crisi mondiale economica, la quale ha avuto il suo effetto nel paese; il grande abbassamento delle remittances le quali provenivano dagli immigranti che rifornivano periodicamente di liquidita l'intera economia e anche dall'indebolimento del settore edilizio il quale e stato fino agli anni 2008-2009 l'elemento principale trainante del prodotto interno lordo, la caduta del ritmo di crescita del PIL, dove le stesse imprese non fiduciose hanno paura di prendere prestiti, anche delle procedure più conservative da parte delle banche, che sono esaurite da pochi clienti.

In questo momento il problema dei prestiti con problemi e molto serio nel paese, e per questa ragione trovare i fattori che governano la loro dinamica e di grande importanza. Questi fattori macroeconomici possono essere mantenuti sotto osservazione per impedirne la loro successiva crescita. Per analizzare questi fattori abbiamo usato il modello di regressione lineare multipla, in cui abbiamo considerato come variabili indipendenti il prodotto interno lordo, i prestiti per l'economia, e anche il tasso reale effettivo di cambio. Dopo aver fatto la regressione ci risulta che la crescita dei prestiti per l'economia aumenterebbe i prestiti con problemi. Questa potrebbe essere una ragione plausibile a spiegare l'andamento decrescente dei prestiti con problemi negli ultimi due anni. Pero l'abbassamento dei prestiti per l'economia potrebbe incidere nel lungo periodo sui prestiti con problemi, perché tante imprese senza la possibilità di finanziarsi non contribuirebbero alla crescita del PIL. Se il livello dei prestiti per l'economia si riduce, sarebbero poche le imprese ad operare nel mercato e il prodotto interno lordo avrà un andamento decrescente. Dall'altra parte, l'abbassamento del PIL diminuirà la performance delle imprese e la loro capacita di essere solventi, il quale farebbe sì che il tasso dei prestiti con problemi si alzi. II modello costruito ci suggerisce che la crescita del prodotto interno lordo incide nell'abbassamento dei prestiti con problemi, e quindi il governo può esercitare delle politiche le quali possano stimolare la crescita economica poiché in questo modo inciderebbe positivamente nell'abbassamento dei prestiti con problemi. La Banca d'Albania ha organizzato alcuni incontri con le banche ed il Ministero delle Finanze per trovare una soluzione. Secondo noi il nostro governo dovrebbe farsi carico di una parte dei prestiti con problemi in modo tale da ripulire i bilanci delle banche da questi prestiti, in modo che esse siano più libere ad erogare nuovi prestiti. Questo darebbe un impulso all'economia. Un'altra soluzione potrebbe essere che lo stato svolga il ruolo di garante per l'emissione di nuovi prestiti, per specifici settori di rilevanza strategica, i quali non possono usufruire di questi prestiti solo dalla mancanza di garanzie, che in Albania, e ad un livello del 140 percento dell'ammontare del prestito.

Per analizzare più a fondo la stabilita finanziaria nel paese, abbiamo fatto un stress test per le fluttuazioni dei prestiti con problemi nel paese. Dopo aver fatto lo stress-test secondo i tre scenari ci risulta che il livello dei prestiti con problemi non gode di una grande stabilita dagli shock macroeconomici o alle rigide situazioni economiche, nei casi in qui i scenari erano più vicini alla realtà. Quindi possiamo dire nel caso di una crisi molto profonda finanziaria i suoi effetti sui prestiti con problemi sarebbero maggiori che in altri paesi quali non sono cosi interconnessi con i fattori macroeconomici come l'Italia. La spiegazione per questa situazione e forse il fatto che la nostra economia e ancora ai primi passi nel mondo capitalista e come tale, una situazione appesantita economica ha un grosso effetto nel portafoglio prestito, la quale fa crescere il rischio dei prestiti con problemi. La nostro economia non si alimenta di grossi investimenti poiché nel totale del nostro sistema bancario solo il 50 percento dei suoi attivi sono incentrati sul prestito (Banca d'Albania 2014).

II nostro suggerimento per abbassare il livello dei prestiti con problemi nei confronti delle banche e che dovrebbero investire di più in formazione dello staff, dove la banca d'Albania può avere anche lei un ruolo attraverso diversi corsi di formazione e anche attraverso la sensibilizzazione delle banche con la regolamentazione da lei creata, siccome abbiamo osservato che spesso le banche non conoscono bene queste regolamentazioni. Oltre a questo, la Banca d'Albania nei suoi controlli sul terreno dovrebbe tener conto anche di esaminare il livello professionale degli analisti del credito.

Le banche potrebbero ristrutturare i loro prestiti, perché spesso cambiando le clausole, l'allungamento della vita del prestito, l'abbassamento del tasso d'interesse, il condono delle multe potrebbe far sì che le imprese con problemi tornassero ad essere imprese sane. Non dovrebbe essere nel interesse delle banche, neutralizzare le loro perdite, attraverso il pignoramento delle garanzie da loro tenute, ma far sì che l'impresa possa tornare in condizioni di lavoro. 
Anche se ciò dovrebbe dire un abbassamento delle entrate pianificate. Proprio per questo anche la Banca d'Albania ha cambiato la sua regolamentazione, che il ristrutturamento, quando viene fatto dal cliente prima che questo abbia mostrato questi problemi, non declassando i prestiti in una classe inferiore. Altrettanto le banche possono pulire i loro bilanci dai prestiti persi cosi che possono abbassare l'alto livello dei prestiti con problemi da stimolare cosi i nuovi prestiti per l'economia.

$E$ in fine, un accesa lotta contro la corruzione bancaria dovrebbe essere lo scopo primario di un fronte unito di tutti i fattori interagenti. Poiché e un tumore che logora ogni sistema, per quanto sanno lui possa essere.

\section{References}

Banka e Shqipërisë. (2009). Buletini i Bilancit të Pagesave. Tiranë:

Banka e Shqiperisë, (2007-2013). Raporti Vjetor i Mbikëqyrjes. Tiranë:

Brasliņš, G., Orlovs, A., Braukša, I., \& Bulis, A. (2014). Gdp and lending behaviour: Empirical evidence for Baltic States Economies, Regional Formation and Development Studies, No. 2 (10), ISSN 2029-9370.

Beck, Roland., Jakubik, Petr., Piloiu, Anamaria. (2013). Non-Performing loans what matters in addition to the economic cycle? 1515 (QB-AR-13-012-EN-N (online)).

Bozdo, A., \& Koka, M. (2014). Il sistema Bancario di fronte ai problemi economici. European Center of Sustainable Development, pg. 87100.

Cappiello, L., Kadareja, A., Sørensen, Ch. K., \& Protopapa, M. (2010). Do Bank Loans and Credit standards have an Effect on Output? European central bank working papers, No. 1150, 28.

Clair, R.T. (1992). Loan Growth and Loan Quality: Some Preliminary Evidence from Texas Banks.

Driscoll, J. C. (2004). Does bank lending affect output? Evidence from the U.S. states. Journal of Monetary Economics, Vol. 51, p. 451471.

Fernandez de Lis, S. M. J., \& Jesus, S. (2000). Credit Growth, Problem Loans and Credit Risk Provisioning in Spain. Working Paper No. 0018, Banco de Espana, October.

Ministria e Financave. (2014). Të dhëna për Shqipërinë. Tiranë.

Havrylchyk, O. (2010). A macroeconomic credit risk model for stress testing the South African banking secotr. (21639).

Hoti, I. (2010). Impakti i ekspozimit në rrezik i industrisë bankare në Shqipëri. Economicus, Nr. 5 fq. 19-33.

INSTAT. (2014, Maj 23). Ndryshimi i PBB tremujore. Tirane, Albania, Albania.

INSTAT. (2014, Maj 23). VSHB tremujore sipas aktivitetit ekonomik me cmime konstante, vlerat të pa zhveshura nga efektet sezonale. Tirane, Albania, Albania.

Instituti i Statistikave të Shqipërisë. (2005-2013). Norma e Inflacionit sipas Çmimeve të Konsumatorit. Tiranë, Albania.

Keeton, W., \& Morris, Ch. S. (1987). Why Do Banks' Loan Losses Differ? Federal Reserve Bank of Kansas City, Economic Review, May, pp. 3-21.

Khemraj \& Pasha,.K (2005). The determinants of non-performing loans: an econometric case study of Guyana".

Maho, N. (2014, April 30). Kredia në pikiatë, Fullani: Bankat janë konkuruese. Panorama.

Pesola, J. (2001). The role of macroeconomic shocks in banking crises.

Schultz, Th. (1971). Investment in Human Capital: The Role of Education and of Research, New York: Free Press.

Virolainen, K. (2004). Macro stress testing with a macroeconmic credit risk model for Finland.

Vlieghe, G. (2001). Indicators of fragility in the UK corporate sector.

\section{Appendice}

I modelli

Per costruire il modello della volatilità dei prestiti con problemi per l'Albania abbiamo utilizzato il programma di statistica E-views.

La tabella nr. 3 e i grafici nr. 3, 4 e 5, li abbiamo costruiti basandosi sulla base dei dati generati da questo programma. Nella tabella più in basso si presentano i risultati del modello.

Tabella 5. II modello di volatilità dei prestiti con problemi in Albania

Dependent Variable: LOG(NPL)
Method: Least Squares
Date:15/05/15 Time: 08:02
Sample(adjusted): 2008:3 2014:2
Included observations: 26 after adjustment

Variable Coefficient Std. Error t-Statistic Prob. 


\begin{tabular}{lllll}
\hline & & & \\
LOG(GDP(-1)) & $\mathbf{- 1 . 5 9 8 3 2 7}$ & 0.423979 & -2.283190 & $\mathbf{0 . 0 3 2 9}$ \\
LOG(KR(-2)) & $\mathbf{6 . 1 4 8 7 1 3}$ & 1.079850 & 3.581804 & $\mathbf{0 . 0 0 1 8}$ \\
LOG(KRE(-1)) & $\mathbf{3 . 7 0 9 1 5 2}$ & 0.316645 & 9.877661 & $\mathbf{0 . 0 0 0 0}$ \\
& & & \\
R-squared & 0.802209 & Mean dependent var & 10.83673 \\
Adjusted R-squared & 0.865515 & S.D. dependent var & 0.664007 \\
S.E. of regression & 0.243506 & Akaike info criterion & 0.129116 \\
Sum squared resid & 1.245196 & Schëarz criterion & 0.276373 \\
Log likelihood & 1.450604 & F-statistic & 75.01148 \\
Durbin-Ëatson stat & 2.319685 & Prob(F-statistic) & 0.000000 \\
\hline
\end{tabular}

\section{Elaborazione dati tramite E-views}

I Test per quando riguardano la significatività statistica

In primo luogo e stata studiata l'autocorrelazione del modello per l'Albania. Per questo abbiamo utilizzato il test di BreuschGodfrey LM.

Tabella 6. l'Autocorrelazione per il modello di volatilità dei prestiti con problemi i n Albania

\begin{tabular}{llll}
\multicolumn{3}{l}{ Breusch-Godfrey Serial Correlation LM Test: } & \\
& & & \\
F-statistic & 3.732546 & Prob. F(2,19) & 0.0429 \\
Obs*R-squared & 6.769742 & Prob. Chi-Square(2) & 0.3339 \\
& & & \\
\hline
\end{tabular}

\section{Elaborazione dati tramite E-views}

In secondo luogo e stata studiata l'eteroschedasticita del modello per l'Albania. Per questo abbiamo utilizzato il test di Breusch-PaganGodfre.

Tabella 7. l'eteroschedasticita del modello di volatilità dei prestiti con problemi per l'Albania

$\begin{array}{lccc}\text { Heteroskedasticity Test: Breusch-Pagan-Godfrey } & & \\ & & & \\ \text { F-statistic } & 1.494110 & \text { Prob. F(3,20) } & 0.2466 \\ \text { Obs*R-squared } & 4.394024 & \text { Prob. Chi-Square(3) } & 0.2219 \\ \text { Scaled explained SS } & 2.554178 & \text { Prob. Chi-Square(3) } & 0.4656\end{array}$

\section{Elaborazione dati tramite E-views}

Tabella 8. La dispersione degli residui per il modello di volatilità dei prestiti con problemi in Albania

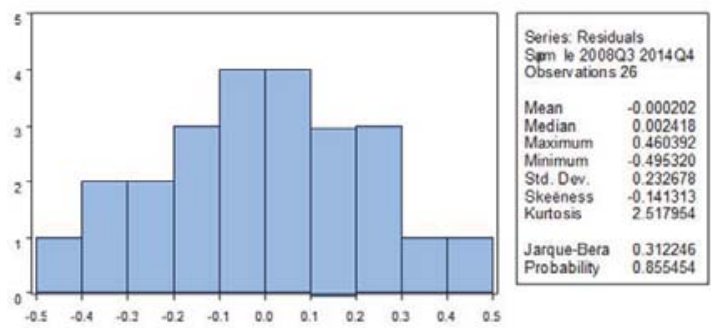

A far concludere, i modelli costruiti sono statisticamente significativi, poiché si passano tutti i test della dispersione normale, eteroschedasticita e dell'Autocorrelazione. 